\title{
Catecholamine Content and Metabolism in the Spontaneously Hypertensive Rats
}

\author{
Masayori Ozaki, M.D.
}

The experiments were primarily carried out with the comparison between normotensive Wistar strain rats (NR) and spontaneously hypertensive rats (SHR).

Tissue catecholamine levels were measured by the trihydroxy indole method, in various tissues at the age of 2 to 3 months, 4 to 6 months, and over 6 months, and only the catecholamine levels of the adrenal gland in SHR were higher than those of NR at the over 4 months of age. Recently Yamori et al reported that the norepinephrine (NR) content in the brain stem of the SHR was significantly lower than NR (N.I.H. Wistar strain). However, the author could not find such differences.

In NR the extraction of the pineal body at 3 weeks of age causes a little higher blood pressure than the nonoperated control rats at the 2 months of age, while SHR showed no noticeable changes.

When 5 hydroxy tryptophan or histidine $(100 \mathrm{mg} / \mathrm{Kg})$ was injected i.p. into the rats, the serotonin $(5 \mathrm{HT})$ or histamine (Hist) content in the brain increased to $40-50 \%$ after 2 to 3 hours. In order to clarify the interreaction between these 2 amines and NE concentration in the brain, we measured the NE content when the $5 \mathrm{HT}$ or Hist increased 2 to 3 hours after the administration of each of the precursors, and found that when $5 \mathrm{HT}$ increased NE decreased, while Hist increased the NE also increased simultaneously. Therefore, there might be some possible roles in these 3 kinds of amines which regulate each other in the brain.

When the urethane was given to the NR, 2 hours later the blood pressure fell from $130-140 \mathrm{mmHg}$ to $100-110 \mathrm{mmHg}$, and the NE content in the brain stem decreased about 20 to $30 \%$. However, when blood presure was regulated by the compensator, ${ }^{*}$ it did not decrease even after urethane anesthesia. In addition the $\mathrm{NE}$ content in the brain stem did not change as that in the brain stem without blood pressure regulation. This is the only preliminary report of the blood pressure compensator, and further investigation will be necessary for the clarification of its activity.

\footnotetext{
From the Department of Pharmacology, Nagoya City University Medical School, Nagoya.

* The compensator has been devised by the author so as to regulate through changing blood volume.
} 